\title{
The influence of scopolamine on motor control and attentional processes
}

Emma Bestaven, Charline Kambrun, Dominique Guehl, Jean-René Cazalets, Etienne Guillaud

Background. Motion sickness may be caused by a sensory conflict between the visual and the vestibular systems. Scopolamine, known to be the most effective therapy to control the vegetative symptoms of motion sickness, acts on the vestibular nucleus and potentially the vestibulospinal pathway, which may affect balance and motor tasks requiring both attentional process and motor balance. The aim of this study was to explore the effect of scopolamine on motor control and attentional processes. Methods. Seven subjects were evaluated on four different tasks before and after a subcutaneous injection of scopolamine $(0.2 \mathrm{mg})$ : a one-minute balance test, a subjective visual vertical test, a pointing task and a galvanic vestibular stimulation with EMG recordings. Results. The results showed that the reaction time and the movement duration were not modified after the injection of scopolamine. However, there was an increase in the center of pressure displacement during the balance test, a decrease in EMG muscle response after galvanic vestibular stimulation and an alteration in the perception of verticality. Discussion. These results confirm that low doses of scopolamine such as those prescribed to avoid motion sickness have no effect on attentional processes, but that it is essential to consider the responsiveness of each subject. However, scopolamine did affect postural control and the perception of verticality. In conclusion, the use of scopolamine to prevent motion sickness must be considered carefully because it could increase imbalances in situations when individuals are already at risk of falling (e.g., sailing, parabolic flight). 
THE INFLUENCE OF SCOPOLAMINE

Emma BESTAVEN 1, Charline KAMBRUN ${ }^{1}$, Dominique GUEHL ${ }^{2,3}$, Jean-René CAZALETS

1, Etienne GUILLAUD ${ }^{1}$

1 Université de Bordeaux, Institut de Neurosciences Cognitives et Intégratives d'Aquitaine, Unité Mixte de Recherche 5287 CNRS, Bordeaux, France.

2 Université de Bordeaux, Institut des Maladies Neurodégénératives, UMR 5293 CNRS, F33000 Bordeaux, France

3 CHU de Bordeaux, Service d'explorations fonctionnelles du système nerveux, F-33000

17 Bordeaux, France

18

19

\section{Corresponding author:}

21 Etienne GUILLAUD, CNRS INCIA UMR 5287, Université Bordeaux Segalen, Zone nord Bât 2 , 22 2e étage, 146 rue Léo Saignat, 33076 Bordeaux cedex France Telephone: +33 5.57.57.16.37 Fax:

$23+335.56 .90 .14 .21$

24 E mail address etienne.guillaud@u-bordeaux.fr

25 


\section{ABSTRACT}

27 Background. Motion sickness may be caused by a sensory conflict between the visual and the 28 vestibular systems. Scopolamine, known to be the most effective therapy to control the 29 vegetative symptoms of motion sickness, acts on the vestibular nucleus and potentially the 30 vestibulospinal pathway, which may affect balance and motor tasks requiring both attentional 31 process and motor balance. The aim of this study was to explore the effect of scopolamine on 32 motor control and attentional processes.

33 Methods. Seven subjects were evaluated on four different tasks before and after a subcutaneous 34 injection of scopolamine (0.2 mg): a one-minute balance test, a subjective visual vertical test, a 35 pointing task and a galvanic vestibular stimulation with EMG recordings.

36 Results. The results showed that the reaction time and the movement duration were not 37 modified after the injection of scopolamine. However, there was an increase in the center of 38 pressure displacement during the balance test, a decrease in EMG muscle response after galvanic 39 vestibular stimulation and an alteration in the perception of verticality.

40 Discussion. These results confirm that low doses of scopolamine such as those prescribed to 41 avoid motion sickness have no effect on attentional processes, but that it is essential to consider 42 the responsiveness of each subject. However, scopolamine did affect postural control and the 43 perception of verticality. In conclusion, the use of scopolamine to prevent motion sickness must 44 be considered carefully because it could increase imbalances in situations when individuals are 45 already at risk of falling (e.g., sailing, parabolic flight). 


\section{INTRODUCTION}

48

49

50

51

52

53

54

55

56

57 64 (Reason 1978).

65

66

67 and cold sweat.

Approximately 10 to $15 \%$ of the population experiences motion sickness in many situations, including travelling by road, sea, or air. This trouble can also occur during simulations in a virtual environment, cinema or video games ("pseudo motion sickness"). Indeed, during parabolic flight, motion sickness is also a major issue and the proportion of subjects with severe symptoms is dramatically increased in the absence of adequate pharmacological treatment. A study from Golding et al. found a far lower rating of motion-sickness in medicated fliers (Golding 2014) than in non-medicated fliers, and the incidence of vomiting in those medicated was also reduced by half. In all cases, the main symptoms experienced were nausea, vomiting

Neural mechanisms of motion sickness have been well described in the literature, and the sensory conflict theory (Reason 1975) seems to be a primary explanation for understanding this state. According to this theory, motion sickness results from a discrepancy between the information provided by the visual and the vestibular systems (Ramos Reis et al. 2013; Schmal 2013). The motion signals transmitted by the eyes and the vestibular system conflict with each other and with what is expected on the basis of previous interactions with the environment

Behavioral as well as pharmacological therapies have been developed to prevent motion sickness. Behavioral strategies, such as avoiding alcohol or coffee intake before travelling and looking at the horizon when sailing, should be prioritized. However, in some extreme conditions when behavioral methods may not be effective, such as during a heavy swell or parabolic flight in an airplane, taking medication may be required. Currently, scopolamine is the most effective drug in 
70 controlling the vegetative symptoms of motion sickness. Scopolamine is a natural alkaloid that 71 acts as a non-specific competitive antagonist of cholinergic muscarinic receptors. As such, this 72 drug acts on the parasympathetic system (Eisenman 2009), which explains some of the 73 secondary effects (dry eyes, dry mouth, changes in intraocular pressure, blurred vision, and 74 dizziness (Brainard \& Gresham 2014)). In the central nervous system, muscarinic receptors are 75 localized in the cortical and subcortical areas. Besides the dorsal part of the brainstem, the 76 vestibular nuclei (upper, lower and lateral) and the cerebellum were identified as some of the 77 scopolamine binding sites (Eisenman 2009). The action of this drug on the vestibular nuclei 78 suggests that it may also act on motor pathways arising from the vestibular nuclei, in particular 79 the vestibulospinal pathway (Weerts et al. 2015b). Since the vestibulospinal pathway regulates 80 body posture and equilibrium (Uchino \& Kushiro 2011), people who use scopolamine may 81 present altered capacities for fine tuning of motor balance in normal and challenging conditions.

82 Further, because vestibular processing relies on a network of brain areas whose epicenter is 83 located in the Sylvian fissure and surrounding parieto-temporal and retro-insular regions (Lopez 84 et al. 2012), scopolamine may potentially affect sensorimotor and cognitive processes related to 85 the vestibular system such as self-motion perception, perception of the vertical or visual 86 processing related to gravitational cues.

87 The aim of the present study was to determine if scopolamine may differentially affect aspects of 88 motor control and/or attentional processes. To answer this question we used several tests: (I) 89 galvanic vestibular stimulation to examine its effect on balance control and lower limb muscle 90 motor responses (Fitzpatrick \& Day 2004); (II) analysis of postural stability by means of center 91 of pressure measures (Duarte \& Freitas); (III) analysis of subjective visual vertical, which is a 92 test for otolithic output; and (IV) a pointing task that measures reaction time and movement 
93 speed. We hypothesized that the scopolamine will disturb balance capacities and will affect the 94 reaction time.

95

96 MATERIALS AND METHODS

97 Participants

98 Seven subjects participated in the experiment (4 men and 3 women, mean age 22.3 SD 2.1).

99 None of them reported vestibular or neuromuscular deficits. The subjects gave their written 100 informed consent and the procedures were in accordance with the ethical standards of the 101 Declaration of Helsinki. The experiments performed in this study were approved by the ethical 102 research committee (Comité de Protection des Personnes Sud Ouest et Outre-Mer III; 2011103 A00424-37 April 27, 2011).

104

105 Scopolamine administration

106 Scopolamine (Scopolamine Bromhydrate; Cooper, France) was administered by subcutaneous 107 injections (dosage: $0.2 \mathrm{mg}$ ). Subjects were tested before and 30 minutes after scopolamine 108 injection. All tests were performed under supervision of a medical doctor. In a previous study the 109 maximum serum concentration was found to occur $17.5 \mathrm{~min}$ (SD 9.8) after the injection of 0.4 $110 \mathrm{mg}$ of scopolamine (Ebert et al. 1998).

111

112 Tests

113 Subjects passed all tests on the same day. That is why they first carried out the tests in the 114 condition OFF scopolamine and then the tests ON scopolamine. However the order of the tests in 
115 the same condition was randomized to avoid any bias such as fatigue. The mean times between

116 the injection of scopolamine and each test recording are reported in Table 1.

117

118 Pointing task

119 Subjects were seated in front of a touch screen $(1024 \times 768)$ with their finger on a sensor located

120 just in front of their navel. The distance between the sensor ant the screen was $42 \mathrm{~cm}$. When the

121 target (a circle) appeared on the screen, they had to touch it as quickly as possible. We tested 3

122 different target diameters $(10,20$ and $30 \mathrm{~mm})$ with 15 trials for each size. The target position was

123 unpredictable and pseudo-randomized on the entire screen. The reaction time (RT) and the

124 movement duration (MD) were recorded. All of the processes were performed by a Matlab

125 routine (Matlab R2013a, Mathworks, Natick, USA).

126

127 Subjective visual vertical

128 The subjective visual vertical (SVV) was assessed using the synapsis SVV device (Synapsys

129 SA, Marseille, France). In a darkroom, a luminous bar was projected on the wall with a video

130 projector. Angular orientation of the bar was changed from trial to trial. The subject was asked to

131 place this bar in a vertical position. The experimenter moved the bar until the subject told him to

132 stop and recorded the angular difference between the actual vertical and the perceived vertical.

133 For each subject, ten trials were recorded and the mean angle value was reported. For each trial,

134 we calculated the deviation from the vertical as the absolute value of the angle measured.

135 Posturography 
136 Subjects remained in a quiet erect position for 1 minute on a multi-component force platform

137 (AMTI, USA, 50Hz) to record the position of their center of pressure (CoP). Subjects performed

138 one trial with their eyes open and one trial with their eyes closed. The position of the feet was

139 identical for both tests. Posturographic parameters were selected based on the suggestions of

140 Prieto et al. (Prieto et al. 1996). The area of the $95 \%$ confidence ellipse, the mean velocity of the

$141 \mathrm{CoP}$ in the anterior-posterior and in the medial-lateral axis, the standard deviation of the CoP

142 travel distance in the anterior-posterior axis (SD-AP) and in the medial-lateral axis (SD-ML)

143 were calculated with a Matlab routine (MathWorks, Natick, USA).

Reaction of postural muscles following a vestibular stimulation

146 To address the effect of scopolamine on balance adjustments that follow vestibular perturbations, 147 we recorded electromyography (EMG) on bilateral Erector Spinae and Gastrocnemius Medialis 148 during Galvanic Vestibular Stimulation (GVS). To apply GVS, two electrodes were positioned 149 on the mastoid processes (Pals platinum, round, $8 \mathrm{~cm}^{2}$ ). In the binaural configuration (see review 150 by Fitzpatrick and Day (Fitzpatrick \& Day 2004)), passing an electric current of $3 \mathrm{~mA}$ between 151 the electrodes increases the activity of the vestibular part of the vestibulocochlear nerve (VIII) 152 located at the cathode side and decreases its activity on the anode side (Goldberg et al. 1984). 153 This artificial change in vestibular nerves is similar to that produced by sway toward the cathode 154 side (Cathers et al. 2005). In our experiment, subjects were asked to turn their head to the right. 155 Using a bipolar constant current generator (Digitimer DS5, Letchworth Garden, United156 Kingdom), binaural GVS was applied with the cathode on the left side. In this configuration, the 157 subjects experienced an illusion of forward sway (slightly left forward because the head was not 158 strictly turned $90^{\circ}$ to the right). The expected response is backside muscles activation (trunk and 
159 lower limbs extensors) with latencies between 50 to $200 \mathrm{~ms}$ (Britton et al. 1993; Cathers et al. 160 2005; Fitzpatrick et al. 1994).

161 EMG from bilateral medial Gastrocnemius and Erector spinae was recorded at $1000 \mathrm{~Hz}$ during 162 the head-turned balance task using an analogical amplifier (TeleEMG, BTS, Milano, Italy) 163 linked to an ITC-18 A/D card (Heka, Lambrecht, Germany). The same A/D card was used to 164 start the GVS stimulation and record the EMG signals; all of the processes were performed by 165 Matlab R2013a (Mathworks, Natick, USA).

166 Each subject $(\mathrm{n}=7)$ received $60 \mathrm{GVS}$ (bipolar binaural square pulse, $3 \mathrm{~mA}, 170 \mathrm{~ms}$ ) per condition 167 (OFF scopolamine, ON scopolamine) spread over 15 sets. Each set consisted of 4 GVS that 168 occurred with randomized delay (inter-stimulation delays were greater than $5 \mathrm{~s}$ ). Between the 169 trials sets, subjects could move their head and have a break if desired. Because visual cues are 170 known to reduce the GVS response amplitude (Cathers et al. 2005; Lund \& Broberg 1983), 171 subjects were blindfolded.

172 EMG signals were analogically amplified (x1k), numerically high-pass filtered (reverse 173 Butterworth filter at $30 \mathrm{~Hz}$ ), and rectified. For EMG signals from Erector Spinae, GVS artifacts 174 (shifts due to voltage) were removed a posteriori by digitalized data shift compensation. For each 175 subject and each stimulation, a $4 \mathrm{~s}$ range was defined around the GVS start (2s before and $2 \mathrm{~s}$ 176 after). All of the EMG ranges were synchronized and averaged to have one sample of 4 seconds 177 per subject, per muscle and per scopolamine condition. These samples were filtered by low-pass 178 filtering (reverse Butterworth filter at $10 \mathrm{~Hz}$ ), and normalized by the activity measured at the 179 trials beginning (tonic activity of normal standing $=100 \%$ ). The EMG medium latency response 180 to the GVS (ML; (Britton et al. 1993)) were visually identified. The start and end delays were 181 measured, ML EMG area and peak amplitude were computed and submitted to statistics. 
182

183 Statistics

184 The results are presented as the mean and standard deviation (SD). For the posturographic tasks,

185 two-way repeated measures ANOVA was used to test the effect of the condition (Open Eyes vs.

186 Closed Eyes) and of scopolamine (OFF vs. ON). For the pointing task, two-way repeated

187 measures ANOVA was used to test the influence of the target size $(10,20$ and $30 \mathrm{~mm})$ and of

188 scopolamine (OFF vs. ON). For the GVS, three-way repeated measures ANOVA was used to

189 test the influence of muscle (Erector Spinae vs. Gastrocnemieus Medialis), scopolamine (OFF

190 vs. ON), and laterality (left muscle vs. right muscle). Finally for the SVV, paired T-test were

191 used to analyze possible differences between the OFF and ON conditions. Correlations were

192 performed using Spearman's correlation coefficient (Rho).

193 Results were considered statistically significant for $P<0.05$. Statistical analysis was performed 194 with IBM SPSS Statistics Version 20 (IBM Corporation, USA).

195

196 RESULTS

197 Pointing task

198 Figure 1 illustrates the results for the RT and the MD. To assess if there was an effect of practice 199 we correlated the trial number with the variables RT and MD, but did not find, however, any 200 relationship. For the reaction time, the Spearman's correlation coefficient was Rho=-0.07 $201(P=0.2)$ for the OFF condition and $\mathrm{Rho}=0.036(P=0.5)$ for the $\mathrm{ON}$ scopolamine condition.

202 Similar findings were obtained for the movement duration in OFF and ON scopolamine 203 condition (respectively Rho=-0.032 $P=0.6$ and $\mathrm{Rho}=-0.072 P=0.2$ ). 
204 Multivariate analysis showed no effect of scopolamine on the RT (Mean_off 0.341 s, SD 0.01; 205 Mean_oN 0.339 s SD $0.02 ; \mathrm{F}(1,6)=1.048, P=0.345$, Fig. 1A) or on the movement duration 206 (Mean_ofF 0.485 s, SD 0.03; Mean_on 0.540 s, SD 0.05; F(1,6)=1.171, $P=0.321$, Fig. 1B). 207 However, there was a significant effect of the target size; the reaction time was longer for 208 smaller targets (Mean_10 0.353 s, SD 0.05; Mean_20 0.336 s, SD 0.05; Mean_30 0.331 s, SD 0.04; $209 \mathrm{~F}(2,5)=13.971, P=0.009)$.

210 Individual data revealed some disparities, particularly in movement duration. As illustrated in

211 figure $1 \mathrm{C}$, although subject 6 had the expected results in the OFF scopolamine condition, the 212 movement duration results increased substantially (MD_ofF $0.458 \mathrm{~s}$; MD_ON $0.514 \mathrm{~s}$ ) after the 213 injection of the drug.

214

215 Subjective visual vertical

216 All subjects had normal results during the OFF scopolamine condition (range from -1.74 to 1.77 217 degrees) with the pathological limit being fixed to 3 degrees by Synapsys. During the ON 218 scopolamine condition, values ranged from $-2.33^{\circ}$ to $3.18^{\circ}$. The mean deviation to the real 219 vertical (absolute value) was higher in the ON scopolamine condition than in the OFF 220 scopolamine condition (Mean_off $1.06^{\circ}$ SD 0.6, Mean_on $1.59^{\circ}$ SD 0.9, $P=0.047$, Fig. 2A).

221 We tested the trial-to-trial variability (10 trials per subject) and found that the subjects' responses 222 were more scattered after the injection of scopolamine, as the SD of the performance increased 223 (Var_ofF 0.674, Var_ON 0.992; $P=0.01$; Fig. 2B).

225 Postural control (Fig. 3) 
226 We computed the posturographic parameters during 1-minute balance tests with eyes opened

227 then with eyes closed. We observed an effect of the scopolamine on the area of the stabilogram

$228(\mathrm{~F}(1,6)=7,171 ; P=0.037)$. With the eyes open, the mean area was $88.8 \mathrm{~mm}^{2} \mathrm{SD} 47.6$ in the OFF

229 scopolamine condition, and was $127.4 \mathrm{~mm}^{2} \mathrm{SD} 97.5$ in the ON scopolamine condition (Fig. 3A).

230 With the eyes closed, the mean area was $100.4 \mathrm{~mm}^{2} \mathrm{SD} 57.9$ in the OFF scopolamine condition,

231 and was $135.2 \mathrm{~mm}^{2} \mathrm{SD} 43.7$ in the ON scopolamine condition (Fig. 3A).

232 The SD-AP was also significantly larger following the injection of scopolamine $(F(1,6)=57.6$,

$233 P<0.001$; Mean_SD-AP_OFF_EyesClosed 4.9 cm SD 1.5; Mean_SD-AP_on_EyesClosed 5.9 cm SD 0.7;

234 Fig. 3B and 3C). No effect of the scopolamine was observed on the SD_ML or on the mean

235 velocity of the CoP.

236

237 Galvanic vestibular stimulation

238 The most important result from the EMG response to GVS was the effect of scopolamine on the 239 response area $(\mathrm{F}(1,6)=10.39, P<0.05$; Fig. 4 A). From OFF scopolamine to ON scopolamine 240 condition, the ML response area decrease was $32.2 \%$ for the Gastrocnemius and $44 \%$ for the 241 erector spinae (in arbitrary units, Gastroc $_{\mathrm{off}}=62.2, \mathrm{Gastroc}_{\mathrm{on}}=20 ; \mathrm{ES}_{\mathrm{off}}=18.8 ; \mathrm{ES}_{\mathrm{on}}=10.8$; fig. 4B).

242 The "muscle" main effect was also significant (Gastrocnemius vs. Erector Spinae, F(1,6)=10.49, $243 P<0.05$ ), with a larger area for the Gastrocnemius than the ES (52 vs. 14.8 a.u., respectively). 244 Similar effects were observed for the maximal amplitude of the ML response.

245 The primary effect of the muscle was observed in the time to peak $(\mathrm{F}(1,6)=7.97, P<0.05)$, with a 246 maximal EMG reached more quickly in the Erector Spinae than in the Gastrocnemius (130 ms 247 vs. $196 \mathrm{~ms}$ after GVS start, respectively). In addition to the observed effect on time to peak, we 248 observed a similar (but not significant, $\mathrm{F}(1,6)=1.86, P=0.22$ ) tendency on the start time of the 
249 ML response with delays of $79 \mathrm{~ms}$ for the Gastrocnemius vs. $50 \mathrm{~ms}$ for the ES. A shorter delay

250 for the ES than for the Gastrocnemius in ML response initiation has been reported by Ali et al.

251 (2003), with latencies of $60 \mathrm{~ms}$ vs. $85 \mathrm{~ms}$.

252

253 DISCUSSION

254 In the present study, we assessed the effects of scopolamine using a before-after approach on 255 various tasks requiring visuo-motor coordination processes, attention, and balance. Although the 256 lack of placebo control group is a limitation of the study design, we found that $0.2 \mathrm{mg}$ of 257 scopolamine did not affect reaction time and movement duration in a pointing task, while in the 258 tasks that challenged the vestibular system (subjective visual vertical, posturography, 259 vestibulospinal reflex elicited by galvanic stimulation), it elicited significant changes.

260 Several studies (Ellis et al. 2006; Ramos Reis et al. 2013; Simmons et al. 2010; Weerts et al. 261 2015b; Wesnes \& Warburton 1984) have evaluated the effects of various doses of scopolamine 262 on cognitive tasks, and partly contradictory results have been reported, likely related to 263 methodological differences between studies. Despite the low number of subjects which could 264 prevent from detecting subtle changes, we found that a $0.2 \mathrm{mg}$ dose of scopolamine did not affect 265 the reaction time and the movement duration (Fig. 1A and 1B). We used this dose because 266 scopolamine is usually prescribed between 0.05 to $0.2 \mathrm{mg}$ to prevent motion sickness (Golding 267 2014). Our results are consistent with those of Weerts et al. (Weerts et al. 2015b), who did not 268 observe changes in RT in a psychomotor vigilance task with a simple RT (click on a mouse 269 button at visual signal). Similarly, Simmons et al. (Simmons et al. 2010) did not find significant 270 effects on RT in a substitution/delayed recall task. In these two experiments, the subjects inhaled

$2710.4 \mathrm{mg}$ of scopolamine by intranasal spray. Although a direct comparison between intranasal and 
272 subcutaneous scopolamine administration (as in the present study) should be made cautiously, it

273 is likely that the $0.4 \mathrm{mg}$ dose of inhaled scopolamine would be closer to the dose used in this

274 study because subcutaneous delivery results in significantly higher bioavailability and higher rate

275 of absorption. The use of higher doses of scopolamine, however, has been found to have an

276 effect on rapid information processing (Wesnes \& Warburton 1984). In a task of consecutive

277 symbol detection, RT was affected by scopolamine at a dose of $1.2 \mathrm{mg}$, whereas a lower dose of

$2780.6 \mathrm{mg}$ did not result in changes. Noticeably, in our experiment, although we injected the same

279 dose for all of the subjects, a female subject presented an exacerbated response. She not only

280 reported that she felt very drowsy, but there was a major effect of scopolamine on her movement

281 time (see subject 6 on figure 1C). Altogether, this indicates that for highly responsive subjects

282 and/or at higher doses, one might expect a decrease in attention or cognitive process, but with the

283 usually prescribed posology for motion sickness prevention $(0.05 \mathrm{mg}$ to $0.2 \mathrm{mg})$, the vigilance

284 tasks will not be significantly affected.

285 To our knowledge, our study is the first to explore the impact of the scopolamine on human

286 balance performance. When the subjects were requested to remain standing in a stationary

287 position, both the CoP area and the anterior-posterior oscillation amplitude were significantly

288 increased after scopolamine administration (Fig. 3). According to these results, scopolamine

289 prescribers should be vigilant to the elderly about the risks of falls which are already increased

290 by age-related changes in the sensorimotor and neuromuscular systems.

291 However, we found a higher inter-subject variability in the eyes-open condition (see error bar

292 Fig. 3A SD=97.5 ON scopolamine). This higher variability in eyes-open condition could be 293 explained by the perceptual styles of the subjects, with a stronger effect of scopolamine on 294 subjects who use somato-sensory cues preferentially (Isableu \& Vuillerme 2006; Kluzik et al. 
295 2005) than on ones with visual preference (Chiari et al. 2000; Isableu et al. 1997). In the eyes 296 closed condition, in which only somato-sensory (including vestibular) cues were available, the 297 perceptual style would have resulted in lower inter-subject variability. This would be in 298 accordance with a recent study (Weerts et al. 2015b) that provided a direct functional 299 demonstration of the impact of scopolamine on cues that originated from the vestibular semi300 circular canals and the utricles. These authors observed a reduction in the gain of the vestibulo301 ocular reflex and a decrease in the total caloric response after scopolamine administration $(0.4$ 302 mg intranasally). These results could be explained by the binding sites for scopolamine on the 303 vestibular nucleus (Jaju et al. 1970; Jaju \& Wang 1971; Matsuoka et al. 1975; Pyykko et al. 304 1984), potentially in the inner ear end organs (vestibular hair cells) and in the vestibular nerve 305 fibers (Li et al. 2007; Weerts et al. 2015a). Moreover, some authors (Kushiro et al. 2008; Uchino $306 \&$ Kushiro 2011) have suggested that the vestibular projections that reach the lower part of the 307 spinal cord (L3) mostly originate from the posterior semi-circular canal. The vestibular inputs are 308 conveyed by the lateral part of the vestibular nucleus (Matsuoka et al. 1975), and this pathway 309 could be affected by scopolamine from the hair cells to the spinal cord (Woolf 1991).

310 The vestibular vagueness induced by scopolamine also appears to have affected the subjects' 311 precision in the subjective visual vertical task (Fig. 3). After scopolamine injection, the mean 312 deviation between SVV and gravitational vector was increased, as well as the subjects' trial-to313 trial variability. As SVV provides an indirect assessment of the otolithic function, this also 314 suggests a greater difficulty in precisely accounting for the sensory cues required to estimate the 315 vertical. Various studies that tested the role of the vestibular system in orientation in patients 316 with vestibular impairments (Borel et al. 2008) have shown that there was a perceived subjective 317 visual vertical tilted toward the side of the lesion (Friedmann 1970; Vibert \& Hausler 2000). 
318 Thus, with regard to our postural tasks, this lack of precision after scopolamine injection could

319 be likely attributed to an altered vestibular input.

320 The GVS test used in the present study directly challenged the balance control originating from

321 vestibular inputs only (Britton et al. 1993; Fitzpatrick \& Day 2004). Following scopolamine

322 administration, we observed a strong reduction of extensor muscle contraction in response to

323 GVS, which suggests a scopolamine action on vestibulospinal processes. Together with the

324 reported effect on CoP oscillations (fig. 2), these results indicate that scopolamine administration

325 may alter functional body balance capacities, even with the low doses $(0.2 \mathrm{mg})$ used for the

326 motion sickness. Although it is difficult to estimate the induced risk for astronauts after

327 scopolamine absorption (to prevent spatial motion sickness), the balance alteration could have

328 more dramatic consequences for people submitted subjected to gravity under terrestrial

329 conditions, such as sailors or parabolic-flight fliers. Similarly, as the vestibulospinal pathways

330 support a large range of motor behavior, such as arm movement correction during unpredicted

331 body displacement (Blouin et al. 2015; Bresciani et al. 2002; Guillaud et al. 2011), scopolamine

332 could affect the control of reaching and grasping movements.

\section{CONCLUSIONS}

334 In conclusion, the use of scopolamine to prevent motion sickness and spatial motion sickness 335 must be considered carefully. Despite the fact that scopolamine has been described as the most 336 effective for motion sickness, our study presents an effect of scopolamine on the vestibulospinal 337 pathway, even at lower doses. Scopolamine could be the source of imbalance in gravitational 338 environments and clumsy motor acts. These effects could be explained by its action on 339 cholinergic muscarinic receptors in the vestibular system. 


\section{REFERENCES}

341 Blouin J, Bresciani JP, Guillaud E, and Simoneau M. 2015. Prediction in the Vestibular Control of Arm Movements. Multisensory Research 28:487-505.

344

345

346

Borel L, Lopez C, Peruch P, and Lacour M. 2008. Vestibular syndrome: a change in internal spatial representation. Neurophysiol Clin 38:375-389.

Brainard A, and Gresham C. 2014. Prevention and Treatment of Motion Sickness. American Family Physician 90:41-46.

Bresciani JP, Blouin J, Sarlegna F, Bourdin C, Vercher JL, and Gauthier GM. 2002. On-line versus off-line vestibular-evoked control of goal-directed arm movements. Neuroreport 13:1563-1566.

Britton TC, Day BL, Brown P, Rothwell JC, Thompson PD, and Marsden CD. 1993. Postural Electromyographic Responses in the Arm and Leg Following Galvanic Vestibular Stimulation in Man. Experimental Brain Research 94:143-151.

Cathers I, Day BL, and Fitzpatrick RC. 2005. Otolith and canal reflexes in human standing. Journal of Physiology-London 563:229-234.

Chiari L, Bertani A, and Cappello A. 2000. Classification of visual strategies in human postural control by stochastic parameters. Human Movement Science 19:817-842.

Duarte M, and Freitas S. Revision of posturography based on force plate for balance evaluation. Revista Brasileira De Fisioterapia 14:183-192.

Ebert U, Siepmann M, Oertel R, Wesnes KA, and Kirch W. 1998. Pharmacokinetics and pharmacodynamics of scopolamine after subcutaneous administration. Journal of Clinical Pharmacology 38:720-726.

Eisenman LM. 2009. Motion sickness may be caused by a neurohumoral action of acetylcholine. Medical Hypotheses 73:790-793.

Ellis JR, Ellis KA, Bartholomeusz CF, Harrison BJ, Wesnes KA, Erskine FF, Vitetta L, and Nathan PJ. 2006. Muscarinic and nicotinic receptors synergistically modulate working memory and attention in humans. International Journal of Neuropsychopharmacology 9:175-189.

Fitzpatrick R, Burke D, and Gandevia SC. 1994. Task-Dependent Reflex Responses and Movement Illusions Evoked by Galvanic Vestibular Stimulation in Standing Humans. Journal of Physiology-London 478:363-372.

Fitzpatrick RC, and Day BL. 2004. Probing the human vestibular system with galvanic stimulation. Journal of Applied Physiology 96:2301-2316.

Friedmann G. 1970. The judgement of the visual vertical and horizontal with peripheral and central vestibular lesions. Brain 93:313-328.

Goldberg JM, Smith CE, and Fernandez C. 1984. Relation between Discharge Regularity and Responses to Externally Applied Galvanic Currents in Vestibular Nerve Afferents of the Squirrel-Monkey. Journal of Neurophysiology 51:1236-1256.

Golding JP, A.; Denise, P. 2014. Prevalence, predictors and prevention of motion sickness in zero-g

Parabolic flights. 6th International congress of medicine in space and extreme environments. Berlin, Germany.

Guillaud E, Simoneau M, and Blouin J. 2011. Prediction of the body rotation-induced torques on the arm during reaching movements: Evidence from a proprioceptively deafferented subject. Neuropsychologia 49:2055-2059. 
401

402

403

404

405

406

407

408

409

410

411

412

413

414

415

416

417

418

419

420

421

422

423

424

425

426

427

428

429

Isableu B, Ohlmann T, Cremieux J, and Amblard B. 1997. Selection of spatial frame of reference and postural control variability. Experimental Brain Research 114:584-589.

Isableu B, and Vuillerme N. 2006. Differential integration of kinaesthetic signals to postural control. Experimental Brain Research 174:763-768.

Jaju BP, Kirsten EB, and Wang SC. 1970. Effects of belladonna alkaloids on vestibular nucleus of cat. American Journal of Physiology 219:1248-\&.

Jaju BP, and Wang SC. 1971. Effects of diphenhydramine and dimenhydrinate on vestibular neuronal activity of cat - a search for locus of their antimotion sickness action. Journal of Pharmacology and Experimental Therapeutics 176:718-\&.

Kluzik J, Horak FB, and Peterka RJ. 2005. Differences in preferred reference frames for postural orientation shown by after-effects of stance on an inclined surface. Exp Brain Res $162: 474-489$.

Kushiro K, Bai R, Kitajima N, Sugita-Kitajima A, and Uchino Y. 2008. Properties and axonal trajectories of posterior semicircular canal nerve-activated vestibulospinal neurons. Experimental Brain Research 191:257-264.

Li J, Chun Y, and Ju H. 2007. Simultaneous electrochemiluminescence detection of anisodamine, atropine, and scopolamine in Flos daturae by capillary electrophoresis using betaCyclodextrin as additive. Electroanalysis 19:1569-1574.

Lopez C, Blanke O, and Mast FW. 2012. The human vestibular cortex revealed by coordinatebased activation likelihood estimation meta-analysis. Neuroscience 212:159-179.

Lund S, and Broberg C. 1983. Effects of Different Head Positions on Postural Sway in Man Induced by a Reproducible Vestibular Error Signal. Acta Physiologica Scandinavica 117:307-309.

Matsuoka I, Domino EF, and Morimoto M. 1975. Effects of cholinergic agonists and antagonists on nucleus vestibularis lateralis unit discharge to vestibular nerve-stimulation in cat. Acta Oto-Laryngologica 80:422-428.

Prieto TE, Myklebust JB, Hoffmann RG, Lovett EG, and Myklebust BM. 1996. Measures of postural steadiness: Differences between healthy young and elderly adults. Ieee Transactions on Biomedical Engineering 43:956-966.

Pyykko I, Schalen L, Jantti V, and Magnusson M. 1984. A reduction of vestibulo-visual integration during transdermally administered scopolamine and dimenhydrinate. A presentation of gain control theory in motion sickness. Acta oto-laryngologica Supplementum 406:167-173.

Ramos Reis PM, Eckhardt H, Denise P, Bodem F, and Lochmann M. 2013. Localization of scopolamine induced electrocortical brain activity changes, in healthy humans at rest. Journal of Clinical Pharmacology 53:619-625.

Reason J. 1975. Motion sickness / J. T. Reason, J. J. Brand. London ; New York: Academic Press.

Reason J. 1978. Motion Sickness - Some Theoretical and Practical Considerations. Applied Ergonomics 9:163-167.

Schmal F. 2013. Neuronal Mechanisms and the Treatment of Motion Sickness. Pharmacology 91:229-241.

Simmons RG, Phillips JB, Lojewski RA, Wang Z, Boyd JL, and Putcha L. 2010. The Efficacy of Low-Dose Intranasal Scopolamine for Motion Sickness. Aviation Space and Environmental Medicine 81:405-412. 
430 Uchino Y, and Kushiro K. 2011. Differences between otolith- and semicircular canal-activated 431 neural circuitry in the vestibular system. Neuroscience Research 71:315-327.

432

433

434

435

436

437

438

439

440

441

442

443

Vibert D, and Hausler R. 2000. Long-term evolution of subjective visual vertical after vestibular neurectomy and labyrinthectomy. Acta Otolaryngol 120:620-622.

Weerts AP, Pattyn N, Putcha L, Hoag SW, Van Ombergen A, Hallgren E, Van de Heyning PH, and Wuyts FL. 2015a. Restricted sedation and absence of cognitive impairments after administration of intranasal scopolamine. $J$ Psychopharmacol.

Weerts AP, Putcha L, Hoag SW, Hallgren E, Van Ombergen A, Van de Heyning PH, and Wuyts FL. 2015b. Intranasal scopolamine affects the semicircular canals centrally and peripherally. Journal of Applied Physiology 119:213-218.

Wesnes K, and Warburton DM. 1984. Effects of scopolamine and nicotine on human rapid information-processing performance. Psychopharmacology 82:147-150.

Woolf NJ. 1991. Cholinergic systems in mammalian brain and spinal-cord. Progress in Neurobiology 37:475-524. 


\section{Table $\mathbf{1}$ (on next page)}

Mean time $(S D)$ between scopolamine injection and task performance 
Mean Time (H:MIN)

Pointing task 01:05 \pm 0:20

Balance task $\quad 00: 56 \pm 0: 26$

SVV $\quad 00: 47 \pm 0: 18$

GVS $\quad 00: 57 \pm 0: 16$

1 
Figure 1 (on next page)

Results of the pointing task

A) Mean reaction time in seconds for each target size $(10,20$ and $30 \mathrm{~mm})$ in both conditions (ON and OFF scopolamine). B) Mean movement duration in seconds for each target size (10, 20 and $30 \mathrm{~mm}$ ) in both conditions ON and OFF scopolamine. C) Movement duration in seconds ON and OFF scopolamine for each subject ( $\mathrm{N}=7$, target size $=20 \mathrm{~mm}$ ). Subject 6 (S6) greatly increased movement duration after the injection of scopolamine. 
A PeerJ
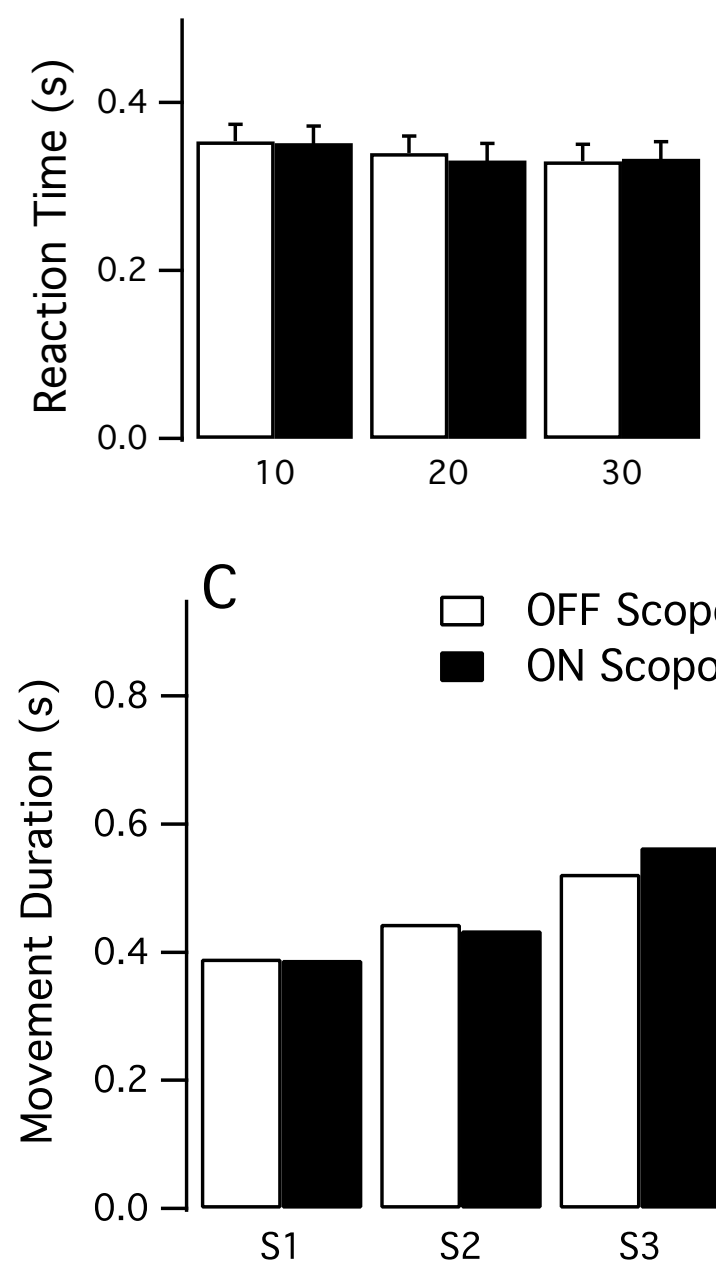

$\square \quad$ OFF Scopolamine

- ON Scopolamine

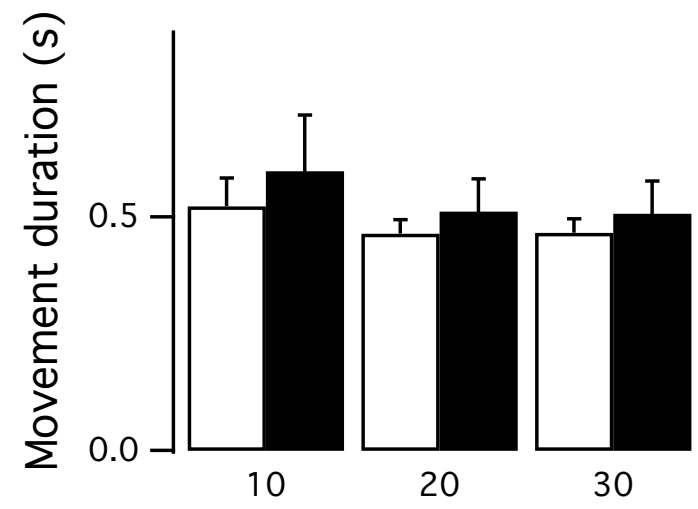

Binuscript to be reviewed 
2

Results of the Subjective Visual Vertical Test

A) Mean deviation from the real vertical in degrees. The deviation significantly increased after the injection of scopolamine $(P<0.05)$. B) Mean trial-to-trial variability (standard deviation) increased in the ON scopolamine condition.
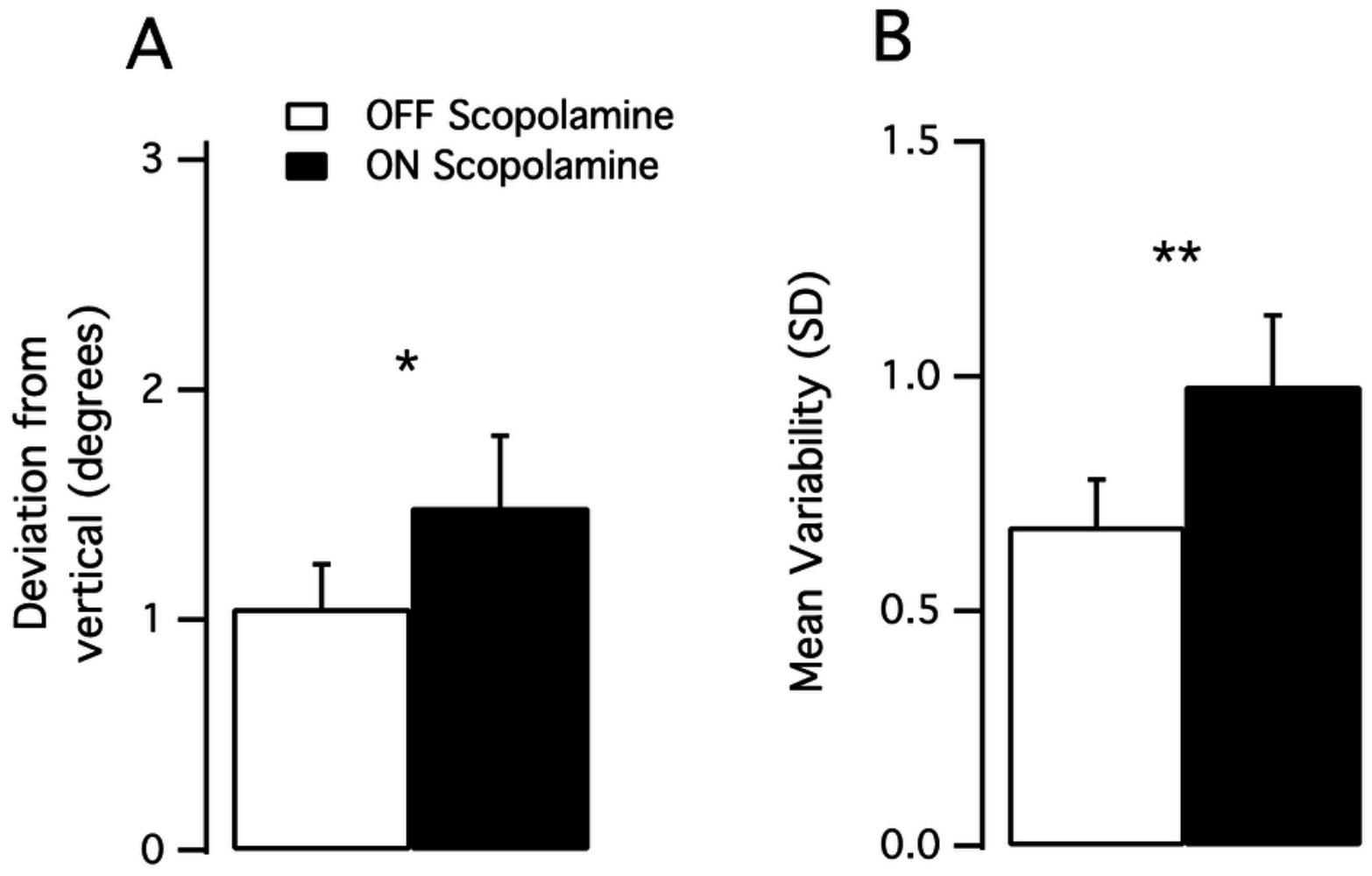


\section{3}

Results of the posturographic tests

A) Mean surface of the stabilogram during a 1-minute balance test with eyes opened (left) then with eyes closed (right). Effect of the scopolamine was significant ( $P=0.037)$. B) The histogram shows the mean standard deviation in the anterior-posterior axis (SD-AP) of the CoP displacement during a 1-minute balance test with eyes closed. C) Stabilograms for a representative subject OFF scopolamine (on the left) and ON scopolamine (on the right) during a 1-minute balance test with eyes closed. The grey curves represent the CoP displacement, and the black curves are the 95\% confidence ellipse.

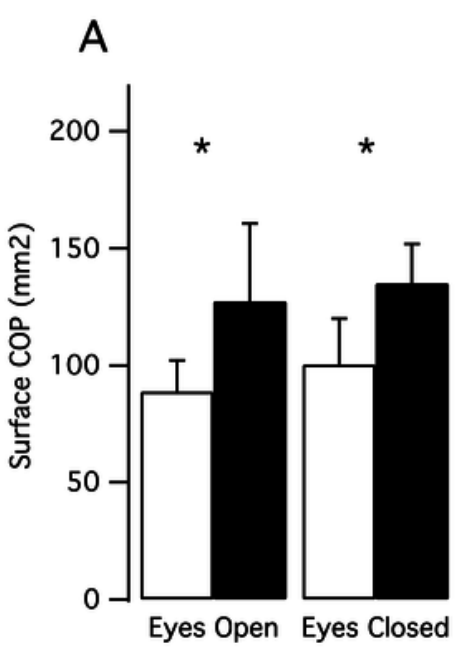

ON Scopolamine

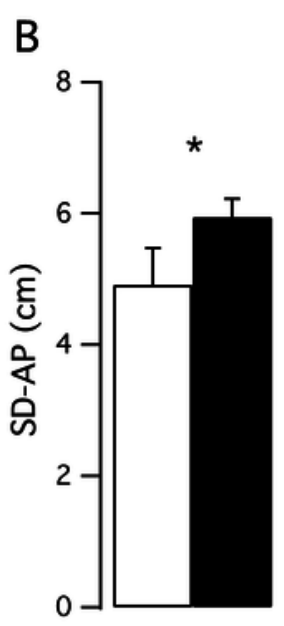

$\square$ OFF Scopolamine

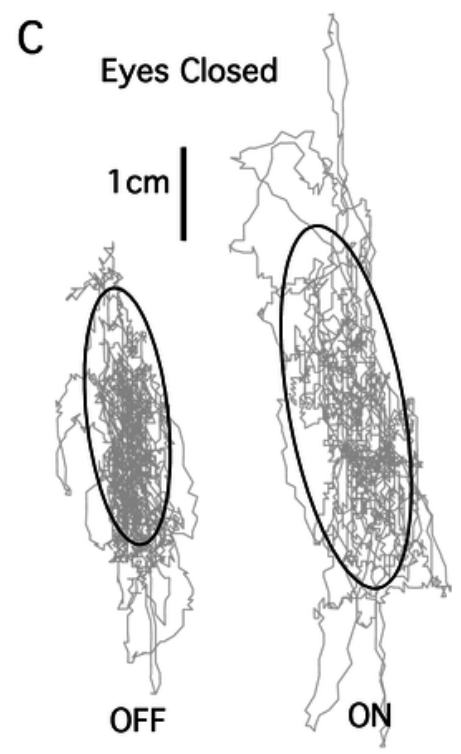


4

Electromyography following GVS

A) Averaged EMG signals from one typical subject in both Gastrocnemius medialis and Erector Spinae. Trials were synchronized on GVS start $(t=0)$, and the muscle activity increased at medium latencies (from 50 to $300 \mathrm{~ms}$ ). In both muscles, scopolamine administration reduced the amplitude of the response. B) Mean EMG response area at medium latency from all subjects. In both muscles, the ML response was reduced in ON scopolamine condition.

A.
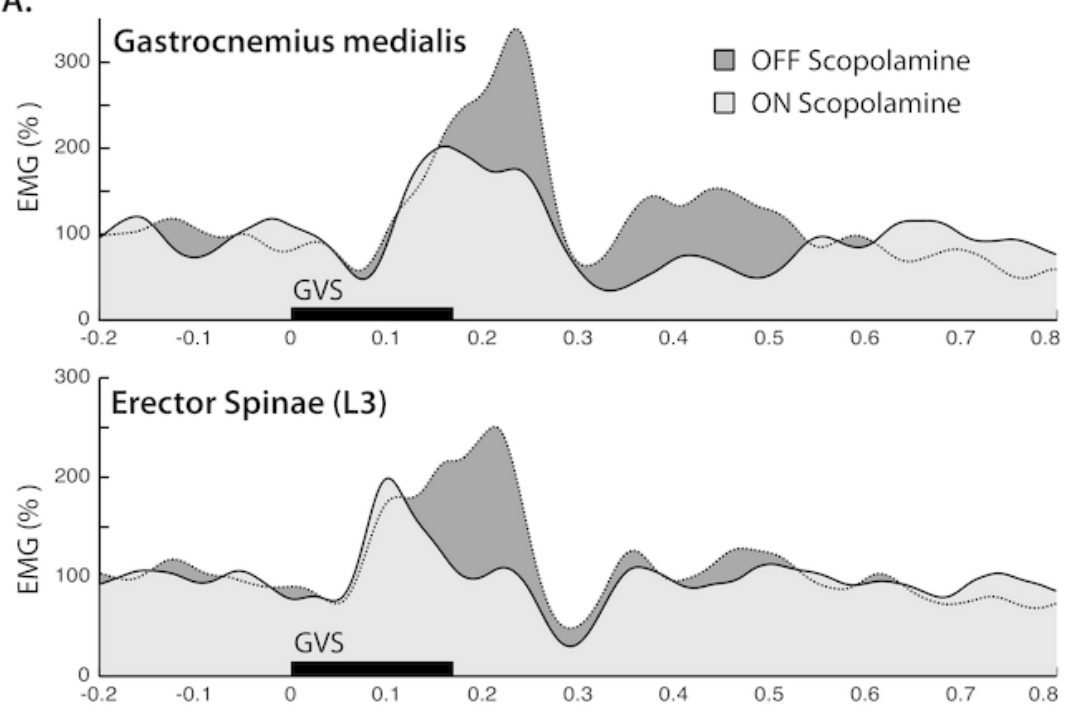

B.

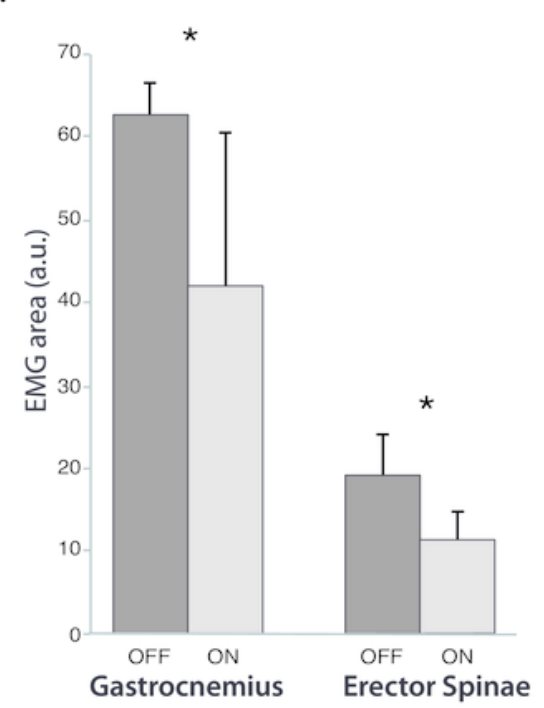

